\section{Praktische Kinder- und Jugendrheumatologie}

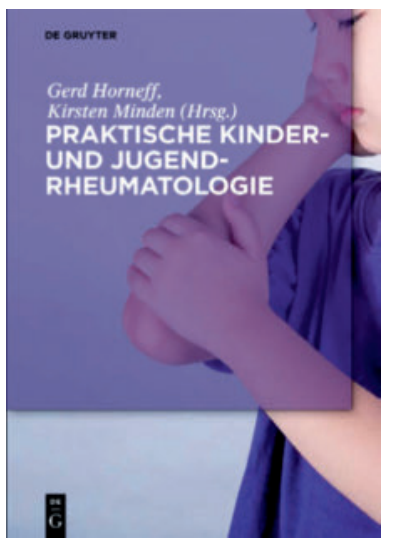

Horneff G, Minden K. Berlin: De Gruyter 2020; Hardcover, 503 S., 167 Farbabbildungen, $38 \mathrm{~s} / \mathrm{w}$ Tabellen, Format $(B \times \mathrm{L}): 17 \times 24 \mathrm{~cm}, 69,95 €$. ISBN 978-3-11-049630-7

Im de Gruyter Verlag ist ein sehr gutes Buch mit dem Titel „Praktische Kinder- und Jugendrheumatologie“, herausgegeben von Kirsten Minden und Gerd Horneff, erschienen. Das Buch erscheint in der 1. Auflage und richtet sich an alle an der Kinder- und Jugendrheumatologie interessierten Kolleginnen und Kollegen. Dabei steht die Vermittlung von praxisrelevantem Wissen im Vordergrund.

Es ist in 2 große Abschnitte gegliedert: Zunächst Grundlagen über klinische Untersuchung und Labordiagnostik sowie allgemeine Aspekte der medikamentösen und nichtmedikamentösen Therapie. In diesem Abschnitt gefällt besonders ein Kapitel über häufige Beschwerden mit Wegweisern zur Diagnosestellung. Dem Kind mit Rückenschmerzen ist ein gesonderter Abschnitt gewidmet. Im 2. Abschnitt werden dann kinder- und jugendrheumatologische Krankheitsbilder kompakt vorgestellt. Neben der klassischen juvenilen idiopathischen Arthritis findet sich hier auch ein eigenes Kapitel über die JIA-assoziierte Uveitis und besonders hervorzuheben ein umfangreiches Kapitel über die Autoinflammatorischen Syndrome, deren Zahl in den letzten Jahren sprunghaft angestiegen ist. Dabei werden auch erst sehr kürzlich be- schriebene Diagnosen wie z. B. COPA-Syndrom erläutert. Hervorragend ist auch der Abschnitt über die seltenen Erkrankungen, die differenzialdiagnostisch ebenfalls zu berücksichtigen sind, wie z. B. die pigmentierte villonoduläre Synovitis oder eine Pachydermodaktylie.

Jedem Kapitel ist die große klinische und didaktische Erfahrung der Herausgeber anzumerken. Auch ganz aktuelle Entwicklungen kommen zur Darstellung, wie z. B. das Treat-to-Target-Konzept und die therapeutische Option der Janus-Kinase-Inhibitoren. Jedes Kapitel schließt ein teilweise umfangreiches, aktuelles Literaturverzeichnis ab.

Abschließend kann gesagt werden, dass das Buch in keiner Kinderklinik fehlen sollte. Es bietet sowohl der/m jungen Assistentin/ en in der Klinik als auch schon erfahrenen Kinder- und Jugendärztinnen und -ärzten kompakte klinische Hilfestellung in der Betreuung und Diagnostik von Kindern und Jugendlichen mit rheumatischen Beschwerden und Krankheitsbildern.

Frank Weller-Heinemann, Bremen

\section{Shoulder Surgery - Tricks of the Trade}

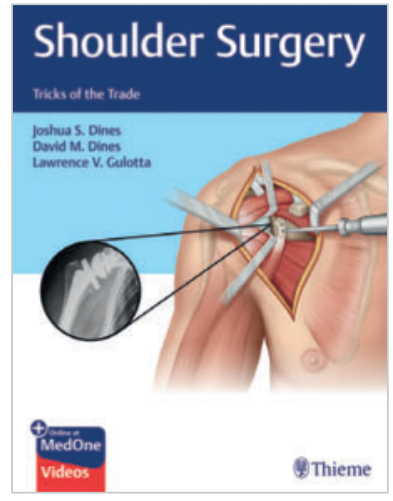

Dines J. S., Dines D. M., Gulotta L. V. Stuttgart, New York: Georg Thieme Verlag 2019; 478 S., 476 Abb., 169,99€. ISBN 978-1-62623-516-8

Die minimal invasive, arthroskopische Chirurgie der Schulter hat in den letzten 20 Jahren einen enormen Aufschwung erfahren und die offenen Operationstechniken bei den meisten Eingriffen an der Schulter mittlerweile als Standardverfahren abgelöst. Dieses Werk besteht aus 73 Kapiteln, in denen zahlreiche, international renommierte Schulterchirurgen unter der Herausgeberschaft der 3 Spezialisten aus dem Hospital for Special Surgery in New York ihre Techniken in der arthroskopischen und offenen Schulterchirurgie sehr strukturiert, präzise und somit leicht nachvollziehbar darstellen. Zahlreiche hervorragende intraoperative Fotos zu den einzelnen Operationsschritten sowie Schemazeichnungen und Bilder von anatomischen Präparaten illustrieren die Textinhalte sehr anschaulich.

Das Buch ist in einem verständlichen Englisch verfasst. Der Text ist darüber hinaus sehr streng gegliedert, sodass auch für den Nicht-Muttersprachler mit guten Englischkenntnissen der Text flüssig zu lesen ist. Insbesondere für Schulterchirurginnen und -chirurgen aus dem europäischen Raum, die nach weiterführenden nützlichen Tricks und Tipps für ihre tägliche Arbeit am Patienten suchen, bietet dieses Werk eine reiche Fundgrube. Neben den arthroskopischen Techniken werden auch offene Techniken, wie z. B. die Muskelersatzoperationen bei Lähmung oder die Endoprothetik des Schultergelenks ausführlich dargestellt. Aus diesem Grund ist das Werk allen erfahrenen Schulterchirurgen mit guten Englischkenntnissen zur Ergänzung ihres operativen Spektrums wärmstens zu empfehlen.

Prof. Dr. Ralph Gaulke, Hannover 